\title{
Tres casos teratológicos en Ligeidos (Heteroptera: Lygaeoidea) chilenos
}

\author{
Three teratological cases on \\ Chilean Lygeids (Heteroptera: Lygaeidae)
}

Eduardo I. Faúndez ${ }^{1,2} \&$ Javiera R. Rocca ${ }^{3}$

\section{Resumen}

Se describen tres casos teratológicos en ejemplares de Lygaeidae de Chile. El primer caso corresponde a una hemiteria pronotal en Syzygitis poecilus (Spinola, 1852); el segundo caso corresponde a una oligomeria simple en la antena derecha de un ejemplar de $S$. poecilus; finalmente el tercer caso corresponde a una atrofia con anartrogénesis en Lygaeus alboornatus Blanchard, 1852. Se comentan y discuten las posibles causas de estas malformaciones.

\section{Palabras clave:}

Teratología, teratosis, Hemiptera, Lygaeinae, Ischnorhynchinae, Chile.

\begin{abstract}
Three teratological cases are described for species in the family Lygaeidae from Chile. The first case belongs to a pronotal hemitery in Syzygitis poecilus (Spinola, 1852). The second case belongs to a simple oligomery in the right antenna of a $S$. poecilus specimen. The third case belongs to an antennal atrophy with anarthrogenesis in Lygaeus alboornatus Blanchard, 1852. The possible causes of these teratosis are commented and discussed.
\end{abstract}

\section{Key words:}

Teratology, teratosis, Hemiptera, Lygaeinae, Ischnorhynchinae, Chile.

\section{INTRODUCCIÓN}

Lygaeidae es una familia de Heteroptera que comprende actualmente 968 especies clasificadas en 102 géneros (Henry, 2009). Las especies de este grupo son principalmente fitófagas; siendo muchas de ellas consideradas de importancia económica (Sweet, 2000). En Chile esta familia cuenta con 14 especies distribuidas desde las regiones de Arica y Parinacota hasta la de Magallanes (Faúndez, 2014); su importancia económica en el país radica en que por lo general hospedan plantas de uso agrícola y sus ninfas por picar semillas para su consumo; mientras que otras especies son de importancia cuarentenaria (Artigas, 1994). Más recientemente, miembros de la subfamilia Lygaeinae han sido detectados dañando plantas de uso ornamental (Faúndez \& Rocca, 2016).

La teratología comprende el estudio de las malformaciones y anormalidades (Carvajal \& Faúndez, 2016). Para Heteroptera, las mayores contribuciones en esta disciplina son las de Balazuc (1951) y Štusak y Stelihk (1977, 1978, 1979, 1980, 1982). Dentro de los heterópteros, los Lygaeoideos son los que presentan el mayor número de reportes (Costas et al. 1992); sin embargo para Chile solo se conoce un caso en Lygaeidae (Faúndez \& Rocca, 2016). El objetivo

1 Entomology Department, School of Natural Resource Sciences, North Dakota State University, Fargo, ND, USA. $\gtrsim$ ed.faundez@gmail.com

2 Departamento de Zoología Médica, Centro de Estudios en Biodiversidad (CEBCh), Magallanes, 1979, Osorno, Chile.

3 Instituto de Biología, Facultad de Ciencias, Pontificia Universidad Católica de Valparaíso, Av. Universidad 330, Placilla (Curauma), Región de Valparaíso, Chile. 
de esta contribución es reportar tres nuevos casos teratológicos para Lygaeidae en Chile.

\section{MATERIALES Y MÉTODOS}

En terminología para la descripción de los casos se sigue a Balazuc (1951) y Štusak \& Stelihk (1977, 1978). En clasificación sistemática de las especies tratadas se sigue a Faúndez (2014). Las fotografías fueron tomadas con una cámara digital adaptada a un microscopio estereoscópico. Todas las medidas se encuentran en milímetros. El material examinado se encuentra depositado en la colección de referencia de heterópteros del Centro de Estudios en Biodiversidad (HRCC).

\section{RESULTADOS}

Caso 1. Hemiteria pronotal en Syzygitis poecilus (Spinola, 1852) (Lygaeidae: Ischnorhynchinae) (Figs. 1-2).

El ejemplar presenta una deformidad pronotal en el ángulo humeral derecho (Fig. 1). Esta estructura se aprecia compactada y ennegrecida, con aspecto tumoral, condición que alcanza incluso el borde anterior derecho del escutelo. En adición, el ángulo humeral derecho del pronoto posee un pequeño tumor ovalado, orientado posteriormente (Fig. 2). En vista dorsal se aprecia un pequeño orificio justo al medio de la zona ennegrecida. La malformación impide cerrar el hemiélitro derecho en posición de reposo.

Material examinado: Chile, Región de Magallanes, Punta Arenas, 28-XI-2008, E. I. Faúndez leg., $1 \widehat{ }$

Caso 2. Oligomeria simple en la antena derecha de Syzygitis poecilus (Spinola, 1852) (Lygaeidae: Ischnorhynchinae) (Fig. 3).

La antena derecha del ejemplar presenta reducción de tamaño por la ausencia del antenómero (Fig. 3). El tercer antenómero (i.e. terminal de la antena malformada) es 0,2 milímetros mas corto que el de la antena normal. La sección distal del tercer antenómero de la antena malformada se encuentra levemente más oscurecido, y con pilosidad similar a la del cuarto antenómero de la antena normal. No se observa anartrogénesis.

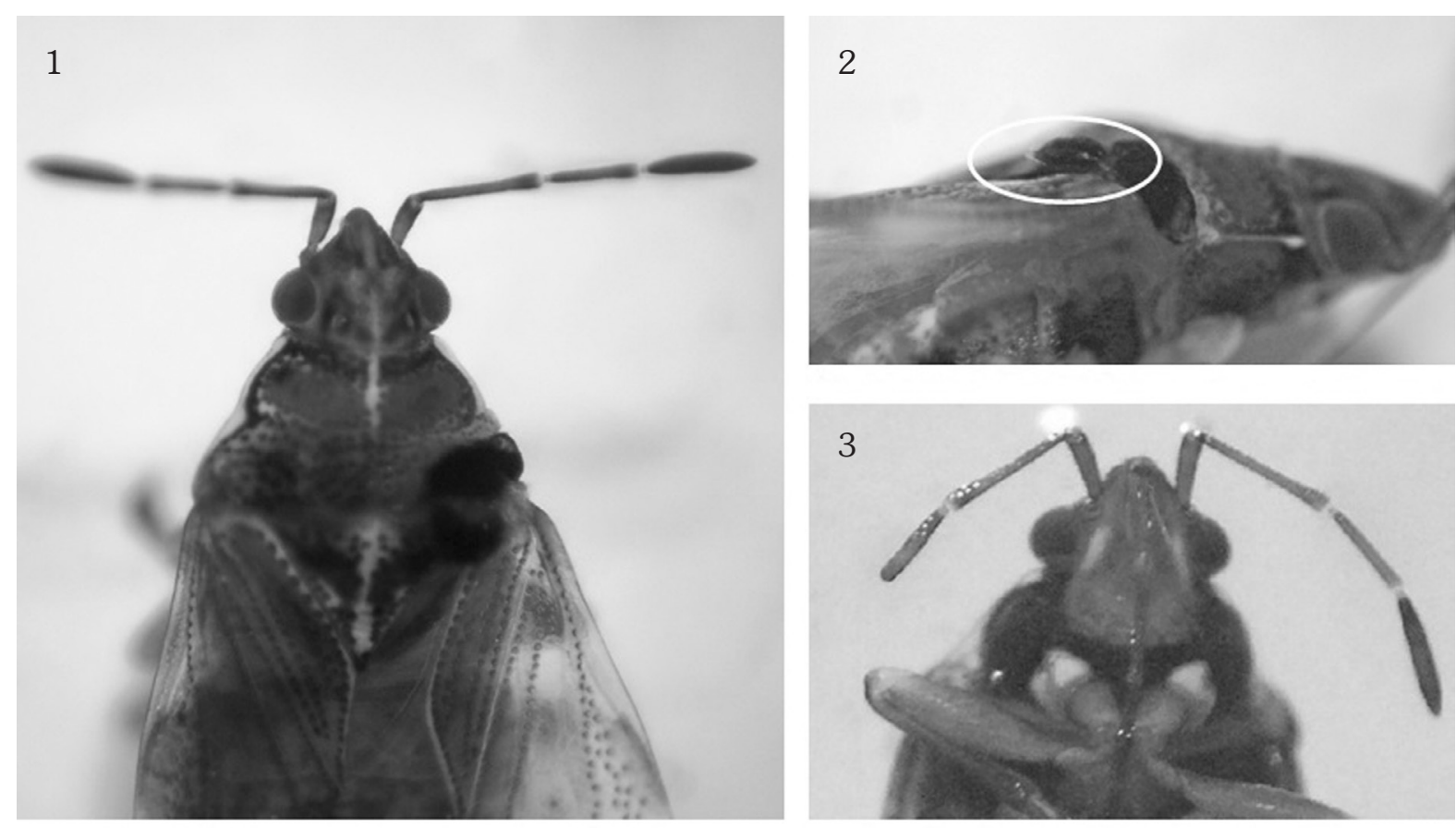

Figs. 1-3. Syzygitis poecilus. Fig. 1. Ejemplar con malformación pronotal, vista dorsal; Fig. 2. Ejemplar con malformación pronotal, vista lateral, tumor resaltado en blanco. 3. Ejemplar con teratosis antenal, vista ventral. 

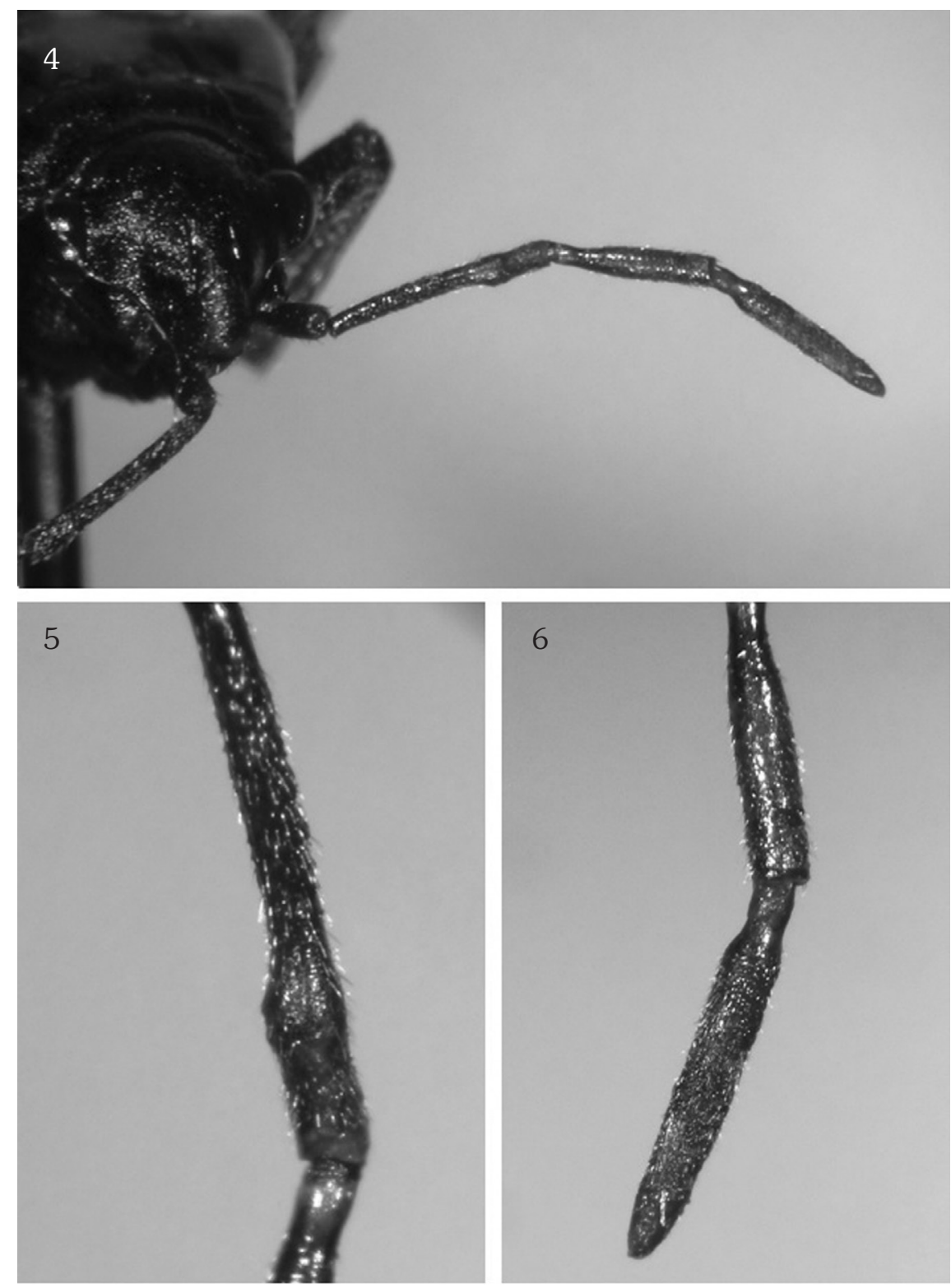

Figs. 4-6. Ejemplar teratológico de Lygaeus alboornatus. Fig. 4. Antena malformada, vista frontal; Fig. 5. Detalle antenómero II y tumor; Fig. 6. Detalle del ápice de la antena malformada.

Material examinado: Chile, Región de Magallanes, Punta Arenas, 11-XII-2007, E. I. Faúndez leg. 1 q.

Caso 3. Atrofia antenal izquierda con anartrogénesis en Lygaeus alboornatus Blanchard, 1852 (Lygaeidae: Lygaeinae) (Figs. 4-6).

La antena izquierda del ejemplar se encuentra atrofiada y con aspecto tumoral (Fig. 4). Pese a que pueden distinguirse segmentos en el pedicelo, estos se encuentran prácticamente fusionados, debido a una esclerotización de la membrana intersegmental (Figs. 4-6). En la sección subapical interna del segundo segmento se aprecia un tumor redondeado convexo (Fig. 5). El último segmento presenta forma y pilosidad normal, excepto en la base que se encuentra esclerotizada y fusionada a lo que debería ser la membrana intersegmental (Fig. 6). A pesar de la antena atrofiada tener las mismas medidas que la antena normal, la proporción de los antenómeros difiere levemente siendo los dos primeros más largos en la antena atrofiada y los 
Tabla 1. Medidas (en mm) de los antenómeros del ejemplar teratológico de L. alboornatus.

\begin{tabular}{lccccc}
\hline Antenómero & I & II & III & IV & Total \\
\hline Antena Izquierda & 0,46 & 1,37 & 0,90 & 1,16 & 3,89 \\
\hline Antena derecha & 0,43 & 1,30 & 0,96 & 1,20 & 3,89 \\
\hline
\end{tabular}

dos restantes más cortos en la misma respecto de la antena normal (Tabla 1$)$.

Material examinado: Chile, Región Metropolitana, Cajón del Maipo, 8-XI-2010, J. Robles leg. 10๋.

\section{DISCUSIÓN Y CONCLUSIONES}

El caso número 1 es la segunda teratosis pronotal descrita en un heteróptero Chileno. Previamente Faúndez y Lüer (2015) reportaron un caso para el pentatómido Oenopiella punctaria (Stål, 1859). El orificio central y el aspecto ennegrecido son típicos de recuperación después de lesiones. Štusak y Stelihk (1977) mencionan que lesiones pronotales en estados ninfales producen teratosis en la familia Tingidae. En este caso, creemos probable que alguna lesión haya ocurrido durante el 5to estado ninfal, ya que la lesión pareciera reciente y probablemente el tumor fue formado por una sección intermedia entre el pronoto y las pterotecas.

El caso número 2 sigue el patrón típico de regeneración después de la pérdida de un antenómero durante algún estado ninfal. Štusak y Stelihk (1978) explican que en estos casos el segmento terminal regenerado puede tomar el aspecto y la pilosidad de un segmento terminal normal, para no perder funciones sensitivas. En este proceso el tamaño de este antenómero puede verse reducido o aumentado; lo que explicaría la leve reducción de tamaño en este caso. La ausencia de anartrogénesis refuerza esta idea, ya que el resto de la antena no se ve afectada en lo absoluto.

El caso número 3 muestra un daño producido por algún agente externo, probablemente debido a una enfermedad ya que el aspecto tumeroso, el tumor del segundo segmento, la anartrogénesis sin una reducción de la antena en tamaño o número de segmentos descarta la amputación; por otro lado una herida local o lateral solo afectaría un área determinada y no el apéndice completo como en este caso. Štusak y Stelihk
(1978) mencionan que en ocasiones enfermedades producidas por patógenos pueden desencadenar teratosis antenales, especialmente atrofias; las que se caracterizan por deformaciones generales $y$ presencia de protuberancias como en el caso aquí descrito.

En Chile, teratosis antenales de heterópteros han sido descritas en el pentatómido Oenopiella punctaria (Stål, 1859) (Faúndez \& Lüer, 2015), en el acantosomátido Ditomotarsus hyadesi Signoret, 1885 (Faúndez \& Carvajal, 2011), en el idiostólido Idiostolus insularis Berg, 1881 (Carvajal \& Faúndez, 2016) y en el ligeido Oncopeltus miles (Blanchard, 1852; Faúndez \& Rocca, 2016); junto con los casos aquí descritos, Lygaeidae se convierte en la familia con mayor cantidad de reportes en el país, liderados por teratosis antenales.

\section{LITERATURA CITADA}

Artigas, J. N. (1994). Entomología Económica. Ediciones Universidad de Concepción, Concepción, Chile, Vol. I, 1126 pp.

Balazuc, J. (1951). La tératologie des Hémiptères et groupes voisins. Annales de la Société Entomologique de France, 120, 17-66

Carvajal, M. A., \& Faúndez, E. I. (2016). A teratological case in the family Idiostolidae (Hemiptera: Heteroptera: Idiostoloidea). Anales del Instituto de la Patagonia 44 (1), 43-46.

Costas, M., López, T., \& Vázquez, M. A. (1992). Teratologías en Lygaeidae (Heteroptera). Actas do $\mathrm{V}$ Congreso Ibérico de Entomología, Lisboa, 1, 313-322.

Faúndez, E. I. (2014). The Lygaeoidea sensu lato of Magallanes Region: Checklist and identification key to the species. Anales del Instituto de la Patagonia, 42(2), 59-63.

Faúndez, E. I., \& Carvajal, M. A. (2011). Un caso teratológico en un ejemplar de Ditomotarsus punctiventris Spinola, 1852 (Hemiptera: Heteroptera: Acanthosomatidae) de 
Magallanes (Chile). Boletín de la Sociedad Entomológica Aragonesa, 48, 431-432.

Faúndez, E. I., \& Lüer, A. (2015). A teratologic case in Oenopiella punctaria (Stål, 1859) (Heteroptera: Pentatomidae: Pentatominae: Carpocorini) from the Aisén region (Chile). Anales del Instituto de la Patagonia, 43(1), 153-156.

Faúndez, E. I., \& Rocca, J. R. (2016). Descripción de un caso teratológico en Oncopeltus (Erythrischius) miles (Blanchard, 1852) (Heteroptera: Lygaeidae) con notas acerca de su distribución y biología. Arquivos Entomolóxicos, 15, 39-43.

Henry, T. J. (2009). Biodiversity of Heteroptera, En R. G. Foottit \& P. H. Adler (Eds.), Insect Biodiversity: Science and Society (pp. 233-263). Oxford: Wiley-Blackwell.

Schuh, R. T., \& Slater, J. A. (1995). True bugs of the World (Hemiptera: Heteroptera): Classification and Natural History. New York: Cornell University Press, Ithaca.

Spinola, M., \& Blanchard, E. (1852). Hemípteros. En C. Gay (Ed.), Historia física y política de Chile. Zoología. (pp. 113-320). Paris. Vol. 7.

Štusak J. M., \& Sthelik, J. L. (1977). First contribution to the teratology of Tingidae
(Heteroptera) reflexion and variability of paranota. Acta Musei Moraviae, Scientiae biologicae, 62, 119-122.

Štusak J. M., \& Sthelik, J. L. (1978). Second contribution to the teratology of Tingidae (Heteroptera) Antennal anomalies. Acta Musei Moraviae, Scientiae biologicae, 63, 89-105.

Štusak J. M., \& Sthelik, J. L. (1979). Third contribution to the teratology of Tingidae (Heteroptera) Anomalies of legs. Acta Musei Moraviae, Scientiae biologicae, 64, 75-84.

Štusak J. M., \& Sthelik, J. L. (1980). Fourth contribution to the teratology of Tingidae (Heteroptera). Anomalies of head and thorax. Acta Musei Moraviae, Scientiae biologicae, 65, 161-172.

Štusak J. M., \& Sthelik, J. L. (1982). Fifth contribution to the teratology of Tingidae (Heteroptera). Anomalies of fore wings (Hemelytra). Acta Musei Moraviae, Scientiae biologicae, 67, 163-180.

Sweet, M. H. (2000). Economic importance of the seedbugs and the chinchbugs (Lygaeoidea) Chapter 6. En C. W., Schaefer \& A R. Panizzi (Eds.), Heteroptera of Economic Importance (pp.143-264). Florida : CRC Press, Boca Raton. 
